Introduction In ARIEL3 (NCT01968213), rucaparib maintenance for recurrent ovarian cancer (rOC) significantly improved investigator-assessed PFS and postprogression efficacy outcomes versus placebo regardless of biomarker status. PFS was also improved in patients with rOC associated with either BRCA1 or BRCA2 mutations (HR, 0.32 [95\% CI, $0.19-0.53]$ and 0.12 [0.06-0.26], respectively). This exploratory analysis further examined the subgroup of patients with rOC associated with BRCA1 or BRCA2 mutations to assess the durability of the clinical benefit of rucaparib maintenance following disease progression.

Methods Patients were randomised 2:1 to oral rucaparib (600 mg twice daily) or placebo. Postprogression efficacy endpoints were assessed in patients with germline or somatic BRCA1 or BRCA2 mutations.

Results Investigator-assessed postprogression efficacy endpoints for patients with either BRCA1 or BRCA2 mutations are presented in the table 1 .

There was a trend for better outcomes across all endpoints in patients with BRCA1 and BRCA2 mutations, with larger differences between the median values among patients with a BRCA2 mutation. The treatment-by-mutation group interaction test reached statistical significance for TFST and CFI.

Among rucaparib-treated patients, the most common treatment-emergent adverse events (any grade) in the BRCA1 and BRCA2 subgroups were nausea $(81.0 \%$ and $78.0 \%)$ and asthenia/fatigue $(74.7 \%$ and $80.0 \%)$.

Conclusions/Implications All postprogression efficacy endpoints were longer with rucaparib maintenance than with placebo in both BRCA-mutant subgroups. Safety data for the two subgroups were similar and were consistent with the overall safety population.
Plenary II

IGCS20_1447

\section{REFINING PATHOLOGIC INTERPRETATION OF ENDOMETRIAL CARCINOMAS: LESSONS LEARNED FROM A NATIONWIDE STUDY IN A NEW ERA OF MOLECULAR CLASSIFICATION}

${ }^{1} \mathrm{E}$ Thompson*, '1 Huvila, ${ }^{2} \mathrm{~S}$ Leung, ${ }^{3} \mathrm{~J}$ Irving, ${ }^{3} \mathrm{~N}$ van der Westhuizen, ${ }^{4} \mathrm{M}$ Kinloch, ${ }^{5} \mathrm{~A}$ Lytwyn, ${ }^{5} \mathrm{M}$ Sur, ${ }^{6} \mathrm{C}$ Parra-Herran, ${ }^{7} \mathrm{~A}$ Yasmeen, ${ }^{8} \mathrm{~F}$ Gougeon, ${ }^{9} \mathrm{C}$ Morin, ${ }^{9} \mathrm{~K}$ Grondin, ${ }^{10} \mathrm{~S}$ Offman, ${ }^{11} \mathrm{~T}$ Salisbury, ${ }^{12} \mathrm{E} \mathrm{He},{ }^{12} \mathrm{~J}$ Lawson, ${ }^{13} \mathrm{~J}$ Vanden Broek, ${ }^{14} \mathrm{C}$ Bell, ${ }^{9} \mathrm{~K}$ Ennour-Idrissi, ${ }^{15} \mathrm{C}$ Wohlmuth, ${ }^{16} \mathrm{D}$ Vicus, ${ }^{16} \mathrm{D}$ Vicus, ${ }^{17} \mathrm{~W}$ Gotlieb, ${ }^{18} \mathrm{~L}$ Helpman, ${ }^{1} \mathrm{~A}$ Lum, ${ }^{1} \mathrm{~J}$ Senz, ${ }^{1} \mathrm{D}$ Huntsman, ${ }^{11} \mathrm{~B}$ Gilks, ${ }^{19} \mathrm{JN}$ McAlpine. ${ }^{1}$ Molecular Oncology, University of British Columbia, Canada; ${ }^{2}$ Genetic Pathology Evaluation Centre, University of British Columbia, Canada; ${ }^{3}$ Pathology and Laboratory Medicine, Royal Jubilee Hospital and the University of British Columbia, Canada; ${ }^{4}$ Pathology and Laboratory Medicine, University of Saskatchewan, Canada; ${ }^{5}$ Pathology and Molecular Medicine, McMaster University, Canada; ${ }^{6}$ Laboratory Medicine and Pathobiology, University of Toronto and Sunnybrook Health Sciences Centre, Canada; 'Gynecologic Oncology, Segal Cancer Center, Jewish General Hospital, McGill University, Canada; ${ }^{8}$ Department of Pathology, University of Montreal, Canada; ${ }^{9}$ Pathology Department, Centre Hospitalier Universitaire de Québec, L'Hôtel-Dieu de Québec, Laval University, Canada; ${ }^{10}$ Anatomical Pathology, Queen Elizabeth II Health Sciences Centre and Dalhousie University, Canada; ${ }^{11}$ Pathology and Laboratory Medicine, Vancouver General Hospital and University of British Columbia, Canada; ${ }^{12} \mathrm{MD}$ Undergraduate Program, University of British Columbia, Canada; ${ }^{13}$ Faculty of Science, University of British Columbia, Canada; ${ }^{14}$ College of Medicine, University of Saskatchewan, Canada; ${ }^{15}$ Gynecologic Oncology, Department of Surgical Oncology, University Health Network, Canada; ${ }^{16}$ Gynecologic Oncology, Centre Hospitalier Universitaire de Québec, L'Hôtel-Dieu de Québec, Canada; ${ }^{17}$ Gynecologic Oncology, Jewish General Hospital, McGill University, Canada; ${ }^{18}$ Gynecologic Oncology, Juravinski Cancer Center and McMaster University, Canada; ${ }^{19}$ Gynecologic Oncology, Vancouver General Hospital and the University of British Columbia, Canada

10.1136/ijgc-2020-IGCS.4

Abstract 4 Table 1 Univariable association of clinicopathologic characteristics by proactive molecular risk classifier for endometrial cancer (ProMisE) subtype

\begin{tabular}{|c|c|c|c|c|c|c|c|c|c|c|c|}
\hline Variable & \multirow{2}{*}{\multicolumn{2}{|c|}{$\begin{array}{c}\text { Total } \\
862\end{array}$}} & \multicolumn{2}{|c|}{ POLE } & \multicolumn{2}{|c|}{ MMR } & \multicolumn{2}{|c|}{ NSMP/p53wt } & \multicolumn{2}{|c|}{ p53abn } & \multirow[t]{2}{*}{ p value } \\
\hline Total & & & 55 & $(6.4 \%)$ & 247 & $(28.7 \%)$ & 387 & (44.9\%) & 173 & $(20.1 \%)$ & \\
\hline Age at dx & & & & & & & & & & & $<0.001$ \\
\hline$\leq 60$ & 321 & $(37.2 \%)$ & 35 & $(63.6 \%)$ & 78 & $(31.6 \%)$ & 181 & (46.8\%) & 27 & $(15.6 \%)$ & \\
\hline$>60$ & 541 & $(62.8 \%)$ & 20 & $(36.4 \%)$ & 169 & $(68.4 \%)$ & 206 & (53.2\%) & 146 & (84.4\%) & \\
\hline $\mathrm{BMI} \geq \mathbf{3 0}$ & 396 & $(45.9 \%)$ & 20 & $(36.4 \%)$ & 117 & $(47.4 \%)$ & 180 & $(46.5 \%)$ & 79 & $(45.7 \%)$ & 0.004 \\
\hline Histotype & & & & & & & & & & & $<0.001$ \\
\hline Endometrioid & 681 & (79.0\%) & 49 & (89.1\%) & 231 & (93.5\%) & 371 & (95.9\%) & 30 & (17.3\%) & \\
\hline$L G$ & 584 & (67.7\%) & 38 & (69.1\%) & 186 & $(75.3 \%)$ & 349 & (90.2\%) & 11 & (6.4\%) & \\
\hline$H G$ & 97 & (11.3\%) & 11 & $(20.0 \%)$ & 45 & $(18.2 \%)$ & 22 & (5.7\%) & 19 & $(11.0 \%)$ & \\
\hline Non endometrioid & 183 & $(21.2 \%)$ & 6 & (10.9\%) & 16 & (6.5\%) & 16 & $(4.1 \%)$ & 143 & (82.7\%) & \\
\hline FIGO stage & & & & & & & & & & & $<0.001$ \\
\hline 1 & 635 & $73.7 \%)$ & 44 & (80.0\%) & 188 & (76.1\%) & 315 & (81.4\%) & 88 & $(50.9 \%)$ & \\
\hline II-IV & 198 & $(23.0 \%)$ & 11 & $(20.0 \%)$ & 52 & (21.1\%) & 56 & (14.5\%) & 79 & $(45.7 \%)$ & \\
\hline LVI & & & & & & & & & & & $<0.001$ \\
\hline positive & 274 & (31.8\%) & 16 & (29.1\%) & 101 & (40.9\%) & 79 & (20.4\%) & 78 & (45.1\%) & \\
\hline negative & 550 & (63.8\%) & 38 & (69.1\%) & 135 & (54.7\%) & 288 & (74.4\%) & 89 & (51.4\%) & \\
\hline $\begin{array}{l}\text { LN sampling } \\
\text { performed } \\
\text { Yes (any) }\end{array}$ & 519 & $(60.2 \%)$ & 36 & (65.5\%) & 155 & $(62.8 \%)$ & 179 & (46.3\%) & 149 & (86.1\%) & \\
\hline LN metastases & & & & & & & & & & & $<0.001$ \\
\hline yes & 93 & (10.8\%) & 5 & (9.1\%) & 22 & (8.9\%) & 22 & (5.7\%) & 44 & $(25.4 \%)$ & \\
\hline $\begin{array}{c}\text { Post-surgical } \\
\text { Treatment } \\
\text { yes }\end{array}$ & 374 & (43.4\%) & 19 & (34.5\%) & 122 & $(49.4 \%)$ & 110 & $(28.4 \%)$ & 123 & (71.1\%) & $<0.001$ \\
\hline
\end{tabular}


Objectives Molecular classification of endometrial carcinoma (EC) enables consistent classification of tumours and provides valuable prognostic and predictive information. Herein we describe molecular subtype distribution and histomorphologic correlates in recently diagnosed (2016) ECs from across Canada.

Methods Molecular classification was performed on representative tumour specimens from participating centres. Clinicopathologic, management and outcome data were collected (REDCap).

Results 1453 ECs from 30 centres have been identified. Complete molecular (ProMisE) and outcome data is reportable for 862 patients. Histologic and clinicopathologic parameters associated with molecular subtype and are summarised in table 1. Amongst participating centres, routine testing of MMR and p53 immunohistochemistry (IHC) was performed in only $23.5 \%$ (range $3.5-80.0 \%$ per centre) and $15 \%(2.2-45.7 \%)$ of cases respectively. We found p53 abn ECs across a range of histotypes, including low grade endometrioid EC. Subclonal p53 staining was observed in 3.9\% of cases and significantly associated with the presence of pathogenic POLE mutations $(\mathrm{p} \leq 0.001)$. Subclonal MMR IHC expression was seen in $3.5 \%$ of cases and has previously been shown to occur predominantly in the context of MLH1 hypermethylation. MMRd was significantly associated with LVI $(\mathrm{p}<0.001)$. ProMisE subtype was significantly associated with clinical outcomes $(p<0.001)$ even in low stage disease [OS $p=0.045$, DSS $p=0.009$, PFS $\mathrm{p}=0.005$ for stage I].

Conclusions Observation of unusual or unexpected p53 and MMR IHC staining patterns and associated clinical implications highlight the importance of routine testing of these parameters in ECs.

\section{IGCS20_1251}

\section{UNDERSTANDING THE CLINICAL IMPLICATION OF MISMATCH REPAIR DEFICIENCY IN ENDOMETRIOID ENDOMETRIAL CANCER THROUGH A PROSPECTIVE STUDY}

${ }^{1} \mathrm{~S}$ Kim* ${ }^{*}{ }^{2} \mathrm{~A}$ Tone, ${ }^{3} \mathrm{~A}$ Pollett, ${ }^{4} \mathrm{R}$ Kim, ${ }^{3} \mathrm{M}$ Cesari, ${ }^{3} \mathrm{~B}$ Clarke, ${ }^{5} \mathrm{~L}$ Eiriksson, ${ }^{6} \mathrm{~T}$ Hart, ${ }^{7} \mathrm{~S}$ Holter, ${ }^{8} \mathrm{~A}$ Lytwyn, ${ }^{9} \mathrm{M}$ Maganti, ${ }^{10} \mathrm{~L}$ Oldfield, ${ }^{10} \mathrm{~T}$ Pugh, ${ }^{11} \mathrm{~S}$ Gallinger, ${ }^{1} \mathrm{M}$ Bernardini, ${ }^{12} \mathrm{~A}$ Oza, ${ }^{3} \mathrm{~V}$ Dube, ${ }^{3} \mathrm{~J}$ Lerner-Ellis, ${ }^{2} \mathrm{E}$ Van de Laar, ${ }^{2} \mathrm{D}$ Vicus, ${ }^{1} \mathrm{~S}$ Ferguson. ${ }^{1}$ Department of Obstetrics and Gynaecology, University of Toronto, Canada; '2Division of Gynecologic Oncology, Princess Margaret Cancer Centre/University Health Network/Sinai Health Systems, Canada; ${ }^{3}$ Department of Laboratory Medicine and Pathobiology, University of Toronto, Canada; ${ }^{4}$ Fred A Litwin Family Centre for Genetic Medicine, University Health Network, Canada; ${ }^{5}$ Division of Gynecologic Oncology, Department of Obstetrics and Gynecology, Juravinski Cancer Centre, McMaster University, Canada; ${ }^{6}$ Department of Psychology, Ryerson University, Canada; 'Zane Cohen Centre for Digestive Diseases, Familial Gastrointestinal Cancer Registry, Mount Sinai Hospital, Canada; ${ }^{8}$ Division of Anatomical Pathology, Department of Pathology and Molecular Medicine, McMaster University, Canada; ${ }^{9}$ Department of Biostatistics, Princess Margaret Cancer Centre/University Health Network, University of Toronto, Canada; ${ }^{10}$ Department of Medical Biophysics, University of Toronto, Canada; ${ }^{11}$ Division of General Surgery, Princess Margaret Cancer Centre/University Health Network/Sinai Health Systems, Canada; ${ }^{12}$ Division of Medical Oncology and Hematology, Princess Margaret Cancer Centre/University Health Network/Sinai Health Systems, Canada

10.1136/ijgc-2020-IGCS.5
Objectives Findings on impact of mismatch repair deficiency (MMRd) on patient outcomes in endometrial cancer (EC) have been inconsistent to date. The objective of this study was to compare oncologic outcomes between MMRd and MMR-intact (MMRi) endometrioid EC (EEC).

Methods Between 2015-2018, we prospectively recruited 668 EC cases from three cancer centers in Ontario, Canada. Tumors were reflexively assessed for MMR protein expression by immunohistochemistry (IHC). Clinicopathological, treatment and survival data were compared between MMRd and MMRi cases.

Results Out of 668, there were 496 EEC (74\%), with 347 MMRi (70\%) and 149 MMRd (30\%) cases treated with surgery and with complete follow-up information. Median follow-up was 16.8 months (6-96 months). MMRd tumors tended to be grade 2 or $3(56 \%$ vs. $29 \%, \mathrm{p}<0.001)$, with propensity for lymphovascular space invasion (LVSI) (29\% vs. $17 \%, \mathrm{p}=0.004)$ and received more adjuvant treatment $(45 \%$ vs. $33 \%, \mathrm{p}=0.03)$. This group also had significantly lower 3 year RFS (78\% vs. $89 \%, \mathrm{p}=0.02)$ although there was no difference in OS $(p=0.91)$. MLH1/PMS2 deficient tumors had the lowest 3-year RFS compared to intact and other MMRd tumors $(76 \%$ vs. $89 \%$ vs $87 \%, \mathrm{p}=0.02)$. After adjusting for age, stage, grade, use of adjuvant treatment, and LVSI status, MLH1/PMS2 deficiency was still associated with the lowest RFS $(p=0.05)$.

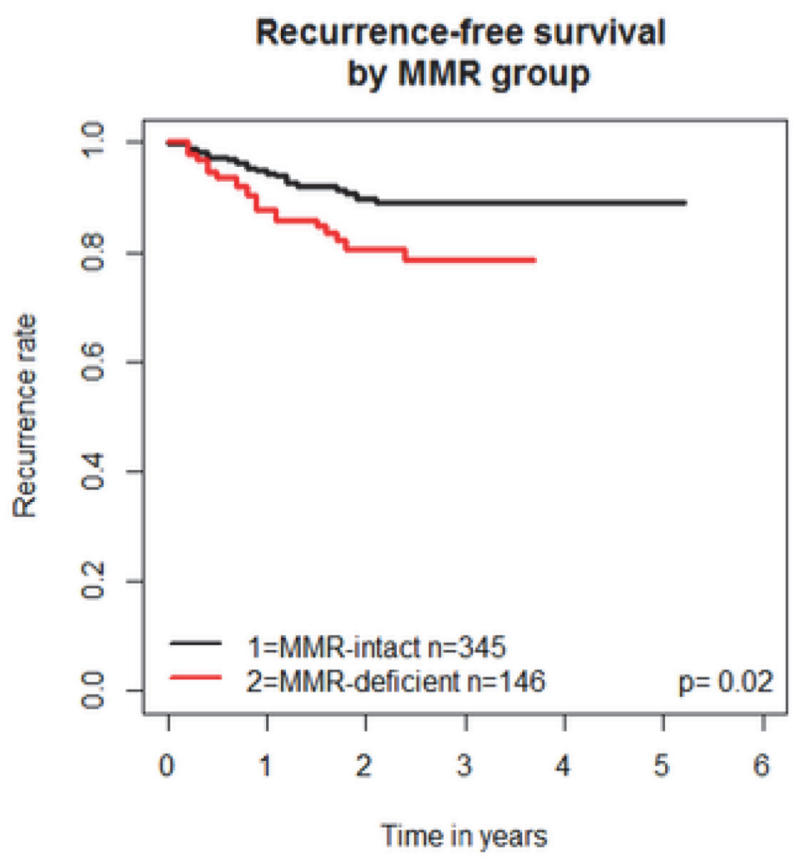

Abstract 5 Figure 1 Comparison of recurrence free survival between mismatch repair deficient (MMRd) and mismatch repair intact (MMRi) endometrioid endometrial cancers

Conclusions MLH1/PMS2 deficient EECs exhibit more aggressive features compared to other MMRd and MMRi cases, with worse RFS. This may indicate an inherent difference in tumor biology, suggesting the importance of individualized management based on tumor's molecular phenotype. 